\title{
Dynamiken des Stillstands
}

\section{Eine sozialwissenschaftliche Perspektive auf kryobiologische Anwendungen}

\author{
Ruzana Liburkina $^{1 *}$, Thomas Lemke ${ }^{1}$, Veit Braun ${ }^{1}$, Sara Lafuente-Funes ${ }^{1}$ \\ ${ }^{1}$ Goethe-Universität Frankfurt am Main, Institut für Soziologie, \\ Theodor-W.-Adorno-Platz 6, 60323 Frankfurt am Main, Deutschland \\ cryosocieties@soz.uni-frankfurt.de \\ * Korrespondenzautorin
}

\section{Kurzfassung}

Gesellschaften der Gegenwart sind vielfach auf Kryotechnologien angewiesen. Sie stellen heute eine wichtige infrastrukturelle Voraussetzung für viele medizinische Anwendungen und einen wesentlichen Motor biowissenschaftlicher Innovationen dar. Zudem eröffnen sie Optionen für individuelle Reproduktionsentscheidungen, ebenso wie für den Erhalt der globalen Biodiversität. Unter der Leitung von Prof. Dr. Thomas Lemke nimmt das Forschungsprojekt CRYOSOCIETIES die Sammlung, Lagerung und Nutzung menschlicher und nicht-menschlicher organischer Materie durch kryobiologische Verfahren aus einer sozialwissenschaftlichen Perspektive in den Blick.

Kryotechnologien rücken in den Fokus gesellschaftswissenschaftlicher Forschung, weil sie grundlegende Normen und Wahrheitsansprüche verschieben. Gleichzeitig steht ihre Anwendung als solche immer im Kontext bestimmter Zukunftsvisionen, Forschungsnetzwerke und Allianzen. Die Untersuchung von Kryokonservierungspraktiken ist somit auch wichtig, um zu verstehen, was heutige Innovationsregime ausmacht und wie ökonomische, politische und epistemische Agenden die Zukunft technologischer Eingriffe in Lebenszyklen prägen.

Dieser Beitrag ordnet diese Erkenntnisinteressen in die Tradition sozialwissenschaftlicher Erforschung von Wissensproduktion und Technologienutzung und -entwicklung ein und skizziert die Potentiale einer solchen Perspektive. Insbesondere werden auch die Besonderheiten eines Studiendesigns diskutiert, das auf unmittelbaren Interaktionen zwischen Sozialwissenschaftler*innen und den Akteur*innen in ihren Forschungsfeldern gründet. Ferner werden die drei Teilprojekte vorgestellt, die dieses empirische Programm in verschiedenen europäischen Staaten und Anwendungsbereichen realisieren.

Die Fallstudien beschäftigen sich mit Nabelschnurblutlagerung, Eizelllagerung und der Kryokonservierung von tierischem Material zum Erhalt der Biodiversität. Dabei stehen jeweils unterschiedliche Forschungsinteressen und -fragen im Mittelpunkt. Sie reichen von Themen, wie Beschäftigungsverhältnissen und Familienplanung über die Verschränkung von Gesundheitsversorgung und Marktstrukturen bis hin zu Mensch-UmweltBeziehungen.

Über diese diversen Felder und Fragestellungen hinweg wird im Rahmen von CRYOSOCIETIES analysiert, wie durch Kryokonservierung neue Zeithorizonte entstehen und wie kryokonservierte Bestände gepflegt, erweitert, verändert und in ihrem Status und Wert verhandelt werden. Diese Untersuchung wirft Fragen auf, die sowohl für Sozialwissenschaftler*innen als auch für Kryoingenieur*innen und -biolog*innen von Interesse sind und Anlässe für Zusammenarbeit und gemeinsame Reflexionsprozesse bieten.

\section{Stichwörter:}

Sozialwissenschaften, Kryobanken, Gesellschaft, Ethnographie, Kryobiologie 


\section{Einleitung}

Am Anfang war Verwunderung. Ein großangelegtes soziologisches Forschungsprojekt, das sich schwerpunktmäßig der Kryokonservierung widmet? Das sei „ungewöhnlich“ oder gar „seltsam“, so die Einschätzung vieler Kryoingenieur*innen und -biolog*innen, denen wir bei unserer Feldforschung begegnet sind. Damit stellt sich die Frage, wie Kryotechnologien zu einem Gegenstand sozialwissenschaftlicher Forschung werden (können): Unter welchen Vorzeichen, mit welchen Methoden und mit welchen Zielsetzungen beschäftigen sich Sozialwissenschaftler*innen mit Kryotechnologien?

In diesem Beitrag skizzieren wir eine Antwort auf diese Frage. Gleichzeitig möchten wir damit auch unsere Erkenntnisinteressen und Forschungsweise gegenüber denjenigen transparent machen, mit deren Arbeit wir uns in unserem Projekt beschäftigen. Wir hoffen, dass unsere Ausführungen dazu beitragen, dass Akteur*innen aus der Forschung, Entwicklung und Anwendung von Kryotechnik systematische Einblicke in die gesellschaftlichen Dimensionen ihrer Arbeit bekommen. Jenseits von Klärung und Reflexion, sollen die nachfolgenden Überlegungen aber auch Denkanstöße für mögliche künftige inter- und transdisziplinäre Formen der Zusammenarbeit liefern.

Wenn hier von ,wir' und ,uns' die Rede ist, handelt es sich in erster Linie um das Autor*innenkollektiv, das zugleich auch das Team der Wissenschaftler*innen ist, die im Rahmen des vom Europäischen Forschungsrat geförderten Projektes CRYOSOCIETIES zusammenarbeiten. ${ }^{1} \mathrm{Im}$ weitesten Sinne gehen wir dabei zwei übergreifenden Fragen nach. Zum einen interessiert uns, wie die Kryokonservierung lebender Materie menschliches (Zusammen)Leben, Wahrheitsansprüche, Zukunftsvisionen und wirtschaftliche Dynamiken beeinflusst. Zum anderen haben wir es uns zum Ziel gesetzt, das Verhältnis zu verstehen, in dem kryobiologische Anwendungen zu den spezifischen politischen, ökonomischen und wissenschaftlichen Problemen unserer Zeit stehen. Um der Vielseitigkeit dieses Feldes gerecht zu werden, richten wir diese Fragen an drei verschiedene Anwendungsgebiete in unterschiedlichen regulatorischen und kulturellen Kontexten: in Spanien die Einlagerung von Eizellen, in Deutschland die Nabelschnurblutkonservierung sowie in Großbritannien die Konservierung von Zell- und genetischen Proben von Tierspezies.

Was uns vier verbindet, ist unser Interesse an und unsere Ausbildung auf einem bestimmten Spezialgebiet der Sozialwissenschaften, nämlich der Wissenschafts- und Technikforschung (Englisch: Science and Technology Studies - STS). Die Entstehung dieses Forschungsgebiets wird auf die Pionierarbeit von Ludwig Fleck in den 1930er und dessen Wiederentdeckung in den 1960er Jahren zurückgeführt. [1] Der Mikrobiologe Fleck zeigte in seinen wissenschaftstheoretischen Schriften, wie die Generierung wissenschaftlichen Wissens unweigerlich in soziale Dynamiken und historische Zusammenhänge eingebettet ist. Einen hohen Bekanntheitsgrad erlangte das Feld dann in den 1980er Jahren, als Soziolog*innen und Sozialanthropolog*innen sich vermehrt auf den Weg in naturwissenschaftliche Labore machten, um Wissensproduktion und Technologieentwicklung im Entstehen zu beobachten. [2] [3] [4] [5]

In den vergangenen dreißig Jahren haben sich die Science and Technology Studies in vielen sozial- und geisteswissenschaftlichen Disziplinen etabliert. Die Palette der Forschungsgebiete und -fragen geht mittlerweile weit über die historische und gesellschaftliche Situierung wissenschaftlicher Fakten hinaus. Wissenschafts- und Technikforscher*innen heute beschäftigen sich unter anderem damit, wie biomedizinische und -technologische Innovationen, Informations- und Kommunikationstechnologien, Klimaforschung und Energieinfrastrukturen die Formen und Bedingungen gesellschaftlichen Zusammenlebens verändern, neuordnen oder aber stabilisieren und verhärten. [6] [7] Sie untersuchen auch, welche gesellschaftlichen Voraussetzungen es überhaupt erst ermöglichen, dass bestimmte Technologien Eingang in Handlungs- und Lebensbereiche finden. In dieser Perspektive entstehen und entwickeln sich Wissenschaft und Technik nicht unabhängig von Gesellschaft; vielmehr bestimmen soziale Kontexte und kulturelle Faktoren, wie sie entstehen, angewendet werden und sich verbreiten. Die Science and Technology Studies sind also das andere, größere ,Wir', auf das sich dieser Beitrag bezieht. Wir interessieren uns für Kryotechnologien, weil gegenwärtige Gesellschaften auf deren Nutzung angewiesen sind. Unser Fokus liegt dabei auf der Sammlung, Lagerung und

\footnotetext{
${ }^{1}$ Die vollständige Bezeichnung des Forschungsprojektes lautet "Suspended Life: Exploring Cryopreservation Practices in Contemporary Societies". CRYOSOCIETIES ist am Institut für Soziologie, Fachbereich Gesellschaftswissenschaften der Goethe-Universität Frankfurt am Main angesiedelt und wird von Prof. Dr. Thomas Lemke geleitet. Die geplante Projektlaufzeit beträgt fünf Jahre: 1. April 2019 bis 31. März 2024.
} 
Aufbereitung von menschlichem und nicht-menschlichem organischem Material durch Verfahren des Kühlens und Gefrierens.

Im Folgenden möchten wir die drei empirischen Fallstudien vorstellen, die das Herzstück des Projekts CRYOSOCIETIES bilden. Dabei werden wir auch unsere Erkenntnisinteressen und Forschungsmethoden erläutern. Insbesondere diskutieren wir die Besonderheiten eines Studiendesigns, das auf unmittelbaren Interaktionen zwischen Sozialwissenschaftler*innen und den Akteur*innen in ihren Forschungsfeldern gründet. Im Schlussteil fassen wir die Potentiale des von uns realisierten Forschungszugangs zusammen. Hier gehen wir sowohl auf die sozialwissenschaftlich signifikanten Erkenntnisgewinne als auch auf die Möglichkeiten ein, welche sich aus unserer Forschungsarbeit für das Untersuchungsgebiet ergeben. Darunter fallen zum einen systematisch aufgearbeitete Reflexionen zu den Auswirkungen von Forschungs- und Entwicklungstätigkeiten und dazu, wie die Ergebnisse dieser Arbeit soziale Dynamiken prägen. Zum anderen gehen wir auf mögliche Kooperationen mit Forschenden und Entscheidungsträger*innen aus dem Feld der Kryobiologie und deren praktischer Anwendung ein. Der Beitrag schließt mit einer Zusammenfassung der Argumentation.

\section{Hauptteil}

Unsere Ausgangsthese ist, dass Kryokonservierung eine spezifische Form des Lebens hervorbringt, die wir als ,Leben in der Schwebe' oder im Englischen als ,suspended life' bezeichnen. [8] Mit diesem Schwebezustand zwischen Leben und Tod, Deanimation und Reanimation gehen neue Möglichkeiten einher, biologische Materie zu kontrollieren, zu modulieren und zu optimieren. Das wiederum hat mitunter weitreichende Effekte auf gesellschaftliche Dynamiken.

Die erste Fallstudie untersucht die Lagerung von Nabelschnurblut für mögliche therapeutische Anwendungen in der Zukunft. Hier hat die Expertise in der Kryokonservierung einer medizinisch notwendigen Substanz schnell Eingang in die Welt kommerzieller Gesundheitsdienstleistungen gefunden. Seit nunmehr mehr als 30 Jahren wird Nabelschnurblut nämlich nicht nur für Zwecke der anonymen Stammzellspende für bestehende schwerwiegende Erkrankungen, sondern auch im Sinne einer privaten Gesundheitsvorsorge eingelagert. Die Praxis der privat finanzierten Einlagerung von stammzellreichhaltigem Nabelschnurblut kann als Antwort auf die wachsende gesellschaftliche Wahrnehmung von Unsicherheit verstanden werden. [9] [10] Die Kosten dafür werden von den Eltern getragen; der tatsächliche Nutzen und das Spektrum der therapeutischen Anwendungsmöglichkeiten für eigenes Nabelschnurblut sind dabei heute nach wie vor umstritten. Nichtsdestotrotz erfreut sich die selbstfinanzierte private Stammzelleinlagerung weiterhin beträchtlicher Beliebtheit. Gleichzeitig sehen sich öffentliche Nabelschnurblutbanken weltweit zum Teil mit massiven Finanzierungsschwierigkeiten konfrontiert, weil die Kosten für die Akquise, Verarbeitung und Lagerung der Kryopräparate die Einnahmen aus tatsächlich abgegebenen Spenden übersteigen. [11] Gerade in Deutschland droht Nabelschnurblutspende zum Auslaufmodell zu werden, obwohl ihr Nutzen (insbesondere) für junge Patient*innen von zahlreichen klinischen Studien belegt wird. [12] Diesem inkongruent anmutenden Verhältnis zwischen biomedizinischer Evidenz und wirtschaftlichem Erfolg geht Ruzana Liburkina hierzulande empirisch nach. In der heutigen Zeit gilt wissenschaftliches Wissen längst nicht mehr als unhinterfragbar oder, in Stein gemeißelt'. Weniger denn je können Entscheidungen mit Gesundheits- und Biomedizinbezug schlicht auf die ,Faktenlage', sondern vielmehr auch auf Hoffnungen und Spekulationen, die schon erwähnten gesellschaftlichen Unsicherheiten und zahlreiche andere Faktoren zurückgeführt werden. Gleichzeitig ist eine starke Verschränkung von Gesundheitsvorsorge und marktwirtschaftlichen Interessen zu beobachten. Die aktuelle Situation im gespaltenen Feld der Nabelschnurblutkonservierung eignet sich sehr gut, um diese gesellschaftlichen Tendenzen besser zu verstehen. Dabei untersucht Liburkina die Strategien, die beide Typen von Nabelschnurblutbanken jeweils mobilisieren, um dem Evidenzproblem beziehungsweise dem Finanzierungsproblem zu begegnen. Im Mittelpunkt steht unter anderem die Frage danach, welchen Wert Kryosammlungen haben und wie sie aufrechterhalten werden, wenn ihre Potentiale zunehmend unklarer werden. Hier spielen die Spezifika solcher Sammlungen eine entscheidende Rolle: Der Betrieb großer Kryobanken ist zwar besonders kostspielig, er schafft aber eine Infrastruktur, die für die Findung und Umsetzung neuer Anwendungsmöglichkeiten genutzt werden können. Außerdem verschafft solche Langzeitlagerung offensichtlich auch Zeit, um die noch unentdeckten Potentiale der konservierten Materie auszuloten. Genau das verfolgt Liburkina im Zuge mehrwöchiger Forschungsaufenthalten unmittelbar in den Banken selbst. Ferner nimmt sie auch die Standpunkte und Erfahrungen von (werdenden) Eltern in den Blick, die die private oder die spendenbasierte Option wählen, um die Stammzellen aus dem Nabelschnurblut ihres Kindes zu konservieren. Schließlich sind es 
ihre Motive und Beweggründe, die über den künftigen Status des Nabelschnurbluts als kryokonservierte Substanz entscheiden.

Die zweite Fallstudie bezieht sich auf die Praxis des sogenannten Social Freezing. Darunter versteht man das ,vorbeugende' oder ,vorsorgliche' Einfrieren von menschlichen Eizellen zur späteren Verwendung für reproduktive Zwecke. Während Eizellen einst vor allem im Fall einer Krebserkrankung und der dafür angewandten zytostatischen und operativen Behandlungsmethoden eingelagert wurden, ist medizinische Notwendigkeit längst nicht mehr das einzige Kriterium, das dabei eine Rolle spielt. Die aufwendig erarbeitete technische Möglichkeit, menschliche Eizellen mittels Vitrifizierung auf lange Sicht konservieren und weitgehend unbeschadet auftauen zu können, wird nun auch vielfach für die selbstgewählte und privat finanzierte Einlagerung genutzt. Diese Möglichkeit, Eizellen ,aufzusparen' und sie in spezifischen Kryobanken zu lagern, wird in den Medien und in der Wissenschaft kontrovers diskutiert. Auf der einen Seite verbindet sich damit die Hoffnung, durch das Einfrieren von Eizellen die Handlungsspielräume für Paare und Frauen zu erhöhen, um berufliche Perspektiven und die Familienplanung leichter miteinander in Einklang zu bringen. Frauen wird damit in Aussicht gestellt, auch in einem höheren Lebensalter auf eingefrorene Eizellen zurückgreifen zu können, um ihren Kinderwunsch zu realisieren. Auf der anderen Seite ist Social Freezing mit körperlichen Belastungen und gesundheitlichen Risiken verbunden. Für die Eizellgewinnung ist eine medizinische Behandlung (unter anderem mit Hormonstimulation und Follikelpunktion) erforderlich und die spätere Nutzung der Eizellen ist nur im Rahmen einer In-vitro-Fertilisation möglich. Kritisch gesehen werden auch die gesellschaftlichen Rahmenbedingungen, auf die diese neue reproduktionsmedizinische Option reagiert. Die Popularität von Social Freezing deutet auf eine Vielzahl von Unsicherheiten hin, mit der sich viele Frauen im reproduktionsfähigen Alter in der heutigen Zeit konfrontiert sehen. [13] Damit interveniert diese Technologie in eine Gemengelage, die gleichzeitig Gegenstand von Gleichstellungs-, Familien- und Sozialpolitik im weitesten Sinne ist. Um genau zu sein, verspricht sie eine technologische und kommerzielle Lösung für weitreichende und kontrovers verhandelte gesellschaftliche Problemstellungen: geschlechtergerechte Beschäftigungs- und Versorgungsstrukturen sowie die Gewährleistung sozialer Sicherheit. Unterdessen entstehen durch die zunehmende Verbreitung von Social Freezing neue Allianzen und Kooperationsformen zwischen Akteuren aus dem Finanzmarkt, Reproduktionsmediziner*innen, Arbeitgebern, Unternehmer*innen und Kryobiolog*innen. [14] Diesen neuartigen Dynamiken und ihren Implikationen für Reproduktionsentscheidungen, Geschlechterrollen, Arbeitsbiographien, Kapitalflüsse und das Verhältnis von Politik und Wirtschaft geht Lafuente-Funes empirisch in Spanien nach. Spanien ist heute nicht nur das wichtigste europäische Land für reproduktionsmedizinische Leistungen, sondern stellt auch für viele Paare aus dem Ausland im Vergleich zu Angeboten aus Kliniken in Osteuropa die attraktivere Alternative dar. Dort führt Lafuente-Funes Interviewgespräche mit Frauen, die ihre Eizellen einlagern lassen. Ferner interviewt sie auch Biolog*innen, Ärzt*innen und Pflegekräfte in mehreren Reproduktionskliniken, wo sie mitunter auch über Wochen als Gastwissenschaftlerin hospitiert.

Das Anwendungsspektrum von Kryobanken ist nicht auf menschliche Körpermaterialien beschränkt, sondern umfasst auch gefrorene Sammlungen von Keimzellen, Gewebe oder DNA von Tier- und Pflanzenarten. In den vergangenen Jahrzehnten hat das beschleunigte Artensterben zu einer ungeheuren Sammel- und Speichertätigkeit geführt, die in den meisten Fällen auf kryotechnischen Verfahren beruht. Diese Kryoprojekte zielen auf den Erhalt der Biodiversität, indem sie seltene oder bereits ausgestorbene Tier- und Pflanzenarten in gefrorener Form konservieren. Dabei werden sehr unterschiedliche Zwecke verfolgt - von der umfassenden Hinterlegung und Nachnutzbarkeit von Forschungsmaterial über die Dokumentation von Zoo- und wildlebenden Tierpopulationen und ihrer Genetik bis hin zur Nutzung von gefrorenen Zellen für die Klonung und Wiederbelebung bedrohter und ausgestorbener Arten in sogenannten Frozen Zoos. [15] [16] All diese Zwecke überschneiden sich; ein und dieselbe Probe kann in sehr unterschiedlichen Kontexten Anwendung finden. Biodiversitätskryobanken lagern daher vor allem präventiv ein, ohne genau zu wissen, welchen Nutzen eine bestimmte Probe in der Zukunft einmal haben wird. Die heute bereits genutzten Proben etwa stammen aus einer Zeit, in der weder genom-weite Untersuchungen noch die Klonung von Tieren möglich waren. Die entsprechenden Verfahren der Kryokonservierung werfen daher die Frage auf, was genau hier bewahrt wird. Durchläuft das zu Bewahrende nicht gerade durch die Praktiken des Einfrierens einen bedeutsamen Transformationsprozess? Kryobanken verschieben die Aufmerksamkeit vom lebenden Organismus auf kleinere (Zellen) und größere (Populationen und Arten) lebende Objekte, die sie zugleich aus ihrem ursprünglichen Zusammenhang lösen und dadurch neu verstehen. In welchem Zustand etwa befindet sich eine Tierart, die seit Jahrzehnten keinen Bezug mehr zu dem Lebensraum hat, in dem sie sich entwickelt hat, und deshalb nicht wieder ausgewildert werden kann? [17] Wird es in Zukunft überhaupt leichter als heute sein, solche Arten 
wieder auszuwildern? [18] Diese Fragen beschäftigen auch die Zoolog*innen, Naturschützer*innen und Biolog*innen, die solche Kryoarchive aufbauen und führen. Die Untersuchung ihrer Arbeit in Frozen Zoos erweitert das Verständnis davon, wie gegenwärtige Gesellschaften die Bewältigung der Biodiversitätskrise angehen und organisieren. Damit ist es ein Feld, das signifikant und exemplarisch ist für die Analyse von Mensch-Umwelt-Beziehungen. Die vielleicht ambitioniertesten solcher Konservierungsbemühungen in Europa finden dabei in Großbritannien statt. In den Konsortien „Frozen Ark" und "CryoArks" - beide koordiniert von der Universität Cardiff - haben sich Zoos, Museen und Forschungslabore zusammengeschlossen. Dort führt Veit Braun teilnehmende Beobachtungen sowie Interviews mit Expert*innen durch. Braun untersucht, wie unterschiedliche Begriffe von Leben, Natur und Zeitlichkeit in diesen Kryokonservierungspraktiken verhandelt und mobilisiert werden und wie die beteiligten Akteur*innen den Nutzen und die Risiken dieser Form von Naturschutz als ,Einlagerung' bewerten.

Die untersuchten Kryobanken enthalten also menschliche ebenso wie nicht-menschliche Proben und dienen medizinischen ebenso wie nicht-medizinischen Anwendungen. Durch die Kombination verschiedener Fallstudien untersucht das Forschungsprojekt Praktiken der Kryokonservierung in ihrer Vielfalt und Komplexität. Die Gesamtprojektleitung, Thomas Lemke, widmet seine Arbeit der Abbildung und der Analyse eben dieser Vielfalt und Komplexität im Rahmen einer übergreifenden sozialtheoretischen Betrachtung des Verhältnisses von Kryotechnologien und gesellschaftlichen Dynamiken.

Über den Fokus auf Kryosammlungen, ihre gesellschaftlichen Entstehungsbedingungen und Implikationen hinaus ist allen drei Teilprojekten von CRYOSOCIETIES der methodologische Zugang gemeinsam. Dieser zielt darauf ab, die Distanz zwischen uns und den Akteur*innen in unseren Forschungsfeldern zu minimieren. So sitzen wir nicht ausschließlich an unseren Schreibtischen und greifen auf sozialwissenschaftliche Theorien zurück, um aus der Ferne über die Dynamiken in den drei Bereichen nachzudenken. Vielmehr begeben wir uns mitten hinein in die Praxisfelder, die wir untersuchen. Die Grundannahme dabei ist, dass sich Dynamiken in gesellschaftlichen Handlungsfeldern am besten verstehen lassen, wenn sie nicht nur aus einer theoretisch versierten Position, sondern auch in Kenntnis der Perspektiven und Praktiken in jenen Feldern erschlossen werden. Konkret heißt das, dass unsere empirischen Untersuchungen darauf abzielen, uns unmittelbar mit der Arbeit und den damit verbundenen Belangen in und rund um Nabelschnurblutbanken, Reproduktionskliniken und Frozen Zoos vertraut zu machen. Dementsprechend sieht unser Untersuchungsdesign nicht etwa vor, dass wir Daten erheben, aggregieren und statistisch auswerten. Stattdessen führen wir zum einen lange Interviewgespräche, welche offen für die Schwerpunktsetzungen und Reflexionen der Interviewten bleiben. Zum anderen verbringen wir viel Zeit in den Organisationen, denen unser Forschungsinteresse gilt. Als Gastwissenschaftler*innen beziehungsweise Hospitant*innen begeben wir uns in die Arbeitsalltage in eben jenen Kryobanken, um die Tätigkeiten, die sie Tag für Tag am Laufen halten, zu beobachten und sich sogar ab und an aktiv an ihnen zu beteiligen.

Diese Herangehensweise hat ihren Ursprung in der Ethnologie (auch bekannt als Kultur- und Sozialanthropologie) und nennt sich daher, ethnographisch'. Dabei geht es in zeitgenössischen ethnographischen Studien meist keineswegs darum, vermeintlich radikal andersartige Gesellschaften aus einer Faszination an dem Fremden heraus zu verstehen. Das Prinzip der Nähe zu den Alltagen und Sichtweisen anderer Menschen ist zwar immer noch Kern ethnographischer Arbeit - auch, wenn nicht auf abgelegenen Pazifikinseln, sondern mitten in Europa in Kryobanken geforscht wird. Nähe zu den Akteur*innen im untersuchten Feld ist im 21. Jahrhundert jedoch mit hohen Ansprüchen an Forschungspartnerschaften verbunden. Als ethnographisch Forschende suchen wir heute Dialog und wechselseitige Lernprozesse. Statt als ,Untersuchungsobjekte“ betrachten wir die Fachkräfte, Expert*innen und Entscheidungsträger*innen vor Ort dabei als essentielle Gesprächspartner*innen, deren Wissen und Standpunkte unsere Erkenntnisproduktion überhaupt erst ermöglichen. [19] [20] Biolog*innen, Ingenieur*innen und Mediziner*innen haben einzigartige Einblicke in und Fragen an die Anwendungsfelder von Kryotechnologien. Diese nachzuvollziehen, einzuordnen und weiterzuverfolgen ist wichtig, um Wissen zu generieren, das auch über sozialwissenschaftliche Fachdiskussionen hinaus von gesellschaftlicher Relevanz ist.

\section{Schlussfolgerungen}

Die Felder, in denen Kryotechnologien für die Konservierung biologischer Materie zur Anwendung kommen, werfen zum Teil sehr diverse Fragen auf. Die drei CRYOSOCIETIES-Fallstudien verdeutlichen, dass je nach 
Anwendungs- und Handlungsgebiet verschiedene Zusammenhänge zwischen Kryotechnologien und heutigen Bedingungen menschlichen (Zusammen)Lebens sichtbar werden. Social Freezing, Nabelschnurbluteinlagerung und Frozen Zoos sind Antworten auf jeweils unterschiedliche Problemstellungen und Herausforderungen, mit denen sich Menschen in Europa und darüber hinaus im 21. Jahrhundert konfrontiert sehen. Indem das Projekt untersucht, wie die Formierung und Entwicklung dieser Felder auf individuelle Handlungsspielräume, soziale Netzwerke, politische Visionen sowie wirtschaftliche Dynamiken einwirken, ermöglicht es eine vielschichtige Einschätzung der sozialen und kulturellen Dimensionen der Schaffung und Aufrechterhaltung kryokonservierten Lebens. Wir analysieren, wie Kryotechnologien nicht nur Zeithorizonte, sondern auch Körperkonzepte und Familienkonstellationen verschieben. Ihr Einsatz verändert Geschlechterverhältnisse und Arbeitsbeziehungen, das Verständnis von Gesundheit und Krankheit, Normalität und Behinderung, Leben und Tod, Nachhaltigkeit und Artenschutz.

Wir gehen von der Annahme aus, dass die Kryokonservierung biologischer Substanzen in allen drei vorgestellten Feldern dazu beiträgt, technologische Lösungen für gesellschaftliche und politische Herausforderungen anzubieten. Im Zuge dessen verändert sie grundlegend, wie Menschen über Zeit und die Zeitlichkeit von Leben denken. Fruchtbarkeit scheint nicht länger mit dem biologischen Alter einer Frau korrelieren zu müssen; wir könnten heute schon unsere Körpersubstanzen für künftige, jetzt noch nicht absehbare medizinische Notfälle und Innovationen bereithalten; selbst ausgestorbene Arten scheinen nicht mehr unwiderruflich der Vergangenheit anzugehören. Die Geradlinigkeit und Unumkehrbarkeit von Lebenszeit und biologischen Zyklen, die lange als unumstrittene Tatsachen galten, sind angesichts der Möglichkeit der Langzeitkonservierung einzelner Körperbestandteile nicht länger selbstverständlich.

Unterdessen rücken die Kryotechnologien selbst selten in den Fokus von Aufmerksamkeit. Auf den ersten Blick bleiben sie unsichtbar - sie scheinen hinter den vielfältigen Anwendungskontexten und innovativen Technologien zu verschwinden, die sie ermöglichen. Unser Anspruch im Rahmen von CRYOSOCIETIES ist es, die Kryotechnologien als infrastrukturelle Voraussetzungen und notwendige Bedingungen sichtbar zu machen und in den Mittelpunkt zu stellen. Sie sorgen erst dafür, dass organische Materie für Biotechnologien zugänglich wird: sei es im Rahmen der In-vitro-Fertilisation, der Gewebezucht oder bei Klonierungsverfahren. Unsere Arbeit wirft Licht auf die praktische und logistische Rolle von Kryosammlungen sowie auf die Funktionen und Netzwerke von Organisationen, die Kryobanken betreiben. Darüber hinaus, macht unsere Forschung greifbar, was in der schwer kategorisierbaren Zwischen- beziehungsweise Übergangszeit passiert, in der biologische Substanzen ,stillgelegt' und für künftige Eingriffe bereitgestellt werden. Während diese Dynamiken häufig unsichtbar bis undurchsichtig bleiben, setzen wir es uns zum Ziel, dieses Wissen nicht nur für ein sozialwissenschaftliches Fachpublikum, sondern auch für diejenigen zur Verfügung und Diskussion zu stellen, die Kryotechnologien entwickeln, optimieren und implementieren. Dieser Beitrag ist Ausdruck dieses Anliegens. Er soll einen Überblick über unsere Forschungsagenda bieten, der Fachkräfte und Forschende aus dem Ingenieurwesen und der Biologie erreicht.

Jenseits der Kommunikation unserer Ziele und grundlegender Thesen möchten wir einige Fragen adressieren, die sowohl für Sozialwissenschaftler*innen als auch für Kryoingenieur*innen und -biolog*innen von Interesse sind und so zu einem gemeinsamen Reflexionsprozess einladen. Dies sind zum einen feldspezifische Fragen:

- Welchen moralischen Status haben kryokonservierte Eizellen? Welche epigenetischen und anderen Unsicherheiten gehen mit dem Einfrieren, Auftauen und der anschließenden intrazytoplasmatischen Spermieninjektion (ICSI) einher?

- Wie unterscheiden sich Stammzell-haltige Substanzen in der Kryokonservierung untereinander sowie von anderen Substanzen, und was lässt sich daraus für die Zukunft von Stammzelltherapien schließen?

- Wie können gefrorene genetische Ressourcen sinnvoll in bestehende Praktiken und Prozesse des Artenschutzes eingebunden werden? Wie verschieben sich Prioritäten in der Biodiversitätsforschung und -bewahrung angesichts wachsender Bestände in Kryobanken?

Zum anderen lohnt es sich auch, feldübergreifende Fragen in interdisziplinären dialogischen Formaten oder gemeinsamen Veröffentlichungen zu verfolgen, wie etwa: 
- Welche neuen Felder der Anwendung von Kryotechnologien könnten in Zukunft noch entstehen? Welche Fragen, Herausforderungen und Chancen für Forschung und Entwicklung in der Kryobiologie werden nicht thematisiert, und warum?

- Wie unterscheidet sich die Zusammenarbeit von Kryoingenieur*innen und -biolog*innen mit diversen Industrie- und Forschungsbereichen (z.B. Stammzellforschung und -banken; Reproduktionsmedizin und -kliniken, Biodiversitätsforschung, Lebensmittelindustrie)?

- Wie haben sich Förderpolitiken, Forschungsprioritäten und Finanzierungsmechanismen für kryobiologische Anwendungsfelder über Zeit entwickelt und wie könnten sie sich in der Zukunft verschieben?

Zu dieser exemplarischen Auswahl können durch den Austausch im Rahmen von Konferenzformaten, wie der Deutsche Kälte- und Klimatagung, noch viele weitere Diskussionsanlässe hinzukommen.

\section{Zusammenfassung}

Kryobiologische Anwendungen reagieren auf gesellschaftliche Dynamiken und beeinflussen diese. Das Forschungsprojekt CRYOSOCIETIES untersucht dieses wechselseitig konstitutive Verhältnis empirisch. Dabei greift es vor allem auf theoretische und analytische Denktraditionen auf dem Gebiet der sozialwissenschaftlichen Wissenschafts- und Technikforschung zurück. Letztere machen es nicht nur möglich, Technologieentwicklung und wissenschaftlichen Wissen in einen historischen und sozialen Zusammenhang zu stellen. Die Ansätze der Science and Technology Studies erlauben es auch, die vielfältigen Effekte zu analysieren, die Wissensproduktion und Technik auf die Formen und Bedingungen gesellschaftlichen Lebens haben. Kryotechnologien sind dabei besonders interessant. Zum einen finden sie in einer Vielzahl an Feldern Anwendung; einige Bereiche zeitgenössischen Lebens sind maßgeblich auf Kryokonservierung angewiesen und wären ohne die entsprechenden Technologien undenkbar. Zum anderen bringen sie eine spezifische und einzigartige Form des Lebens hervor: Organische Materie wird dauerhaft in einen Zwischen- beziehungsweise Übergangszustand zwischen Leben und Tod versetzt. Das schafft neue Zeitverläufe, Handlungsspielräume, soziale Netzwerke und wirtschaftliche und politische Entwicklungen. Statt diese ,Dynamiken des Stillstands' aus Distanz zu beobachten, mobilisiert das Team von Wissenschaftler*innen bei CRYOSOCIETIES ethnographische Erhebungsmethoden. Diese positionieren uns nahe an den Belangen, Herausforderungen und Perspektiven in unseren Untersuchungsfeldern. So arbeiten wir als Gastwissenschaftler*innen/Hospitant*innen mehrere Wochen am Stück in Organisationen, die Kryobanken betreiben, um die Arbeitsalltage dort kennen zu lernen. Außerdem führen wir ausführliche Interviewgespräche mit Menschen durch, die Kryotechnologien entwickeln, kommerziell oder für Forschungszwecke anwendbar machen und für persönliche Zwecke nutzen. Um der Bandbreite der Kryokonservierungspraktiken gerecht zu werden, setzten wir dieses Untersuchungsdesign in insgesamt drei Anwendungsbereichen um: Unsere Fallstudien beschäftigen sich mit der Einlagerung menschlichen Nabelschnurbluts, mit der Vitrifizierung menschlicher Eizellen sowie mit der Kryokonservierung nicht-menschlicher DNA- und Zell-Proben. Dabei stehen jeweils unterschiedliche Forschungsinteressen und fragen im Mittelpunkt. Diese reichen von Themen, wie Beschäftigungsstrukturen und Familienplanung über die Verschränkung von Gesundheitsversorgung und Marktstrukturen bis hin zum Anthropozän und MenschUmwelt Beziehungen. Über diese diversen Felder und Fragestellungen hinweg, interessiert uns, wie durch Kryokonservierung neue Zeithorizonte entstehen und wie kryokonservierte Bestände gepflegt, erweitert, verändert und in ihrem Status und Wert verhandelt werden. Das Wissen, das wir dabei generieren, kann und soll Reflexionsprozesse anregen. Diese können von unseren Annahmen und Einsichten angeregt werden, welche wir systematisch mit Ingenieur*innen und Biolog*innen teilen wollen. Reflexionsprozesse können und sollten im Idealfall aber auch im wechselseitigen Austausch zwischen Sozialwisenschaftler*innen und Expert*innen für Kryotechnologien angestoßen und mit Leben gefüllt werden. In Konferenz- und WorkshopFormaten können und wollen wir Fragen adressieren und neuformulieren, die über disziplinäre Grenzen hinweg von Interesse und Relevanz sind.

\section{Danksagung}

Das Forschungsprojekt "Suspended Life: Exploring Cryopreservation Practices in Contemporary Societies" (CRYOSOCIETIES) wird vom Europäischen Forschungsrat gefördert (ERC Grant Agreement ID 788196). Für die Möglichkeit, das Projekt an dieser Stelle vor- und zur Diskussion zu stellen, gilt unser Dank Prof. Dr. Birgit Glasmacher und der von ihr geleiteten Arbeitsgruppe „Kryokonservierung und Kryotechnik für die regenerative 
Medizin“ am Niedersächsischen Zentrum für Biomedizintechnik, Implantatforschung und Entwicklung sowie am Institut für Mehrphasenprozesse, Leibniz Universität Hannover.

\section{Literaturverzeichnis}

[1] Kuhn, T. S., The Structure of Scientific Revolutions, 1st Edition, University of Chicago Press, Chicago, 1962

[2] Knorr-Cetina, K., The Manufacture of Knowledge: An Essay on the Constructivist and Contextual Nature of Science, 1st Edition, Pergamon Press, Oxford, 1981

[3] Latour, B. \& Woolgar, S., Laboratory Life: The Construction of Scientific Facts, 2nd Edition, Princeton University Press, Princeton, 1986

[4] Lynch, M., Art and Artifact in Laboratory Science: A Study of Shop Work and Shop Talk in a Research Laboratory, 1st Edition, Routledge \& Kegan Paul, London, 1985

[5] Traweek, S., Beamtimes and Lifetimes: The World of High Energy Physicists, 1st Edition, Harvard University Press, Cambridge MA, 1988

[6] Beck, S., Niewöhner, J. \& Sørensen, E. (Hrsg.), Science and Technology Studies. Eine sozialanthropologische Einführung, 1. Auflage, transcript, Bielefeld, 2012

[7] Bauer, S., Heinemann, T., Lemke, T. (Hrsg.), Science and Technology Studies. Klassische Positionen und aktuelle Perspektiven, 1. Auflage, Suhrkamp, Berlin, 2017

[8] Lemke, T., Beyond Life and Death. Investigating Cryopreservation Practices in Contemporary Societies, Soziologie 48 (2019), 450-466

[9] Manzei, A., Stammzellen aus Nabelschnurblut. Gesellschaftliche und ethische Aspekte, 1. Auflage, Institut Mensch Ethik und Wissenschaft (IMEW), Berlin, 2005

[10] Manzei, A., „Nabelschnurblut, die biologische Lebensversicherung für ihr Kind...". Eine gouvernementalitätstheoretische Analyse der Machtverhältnisse in der Biomedizin, in: S. Berghahn, F.O. Wolf \& D.-G. Schulze (Hrsg.), Politisierung und Ent-Politisierung als performative Praxis, Westfälisches Dampfboot, Münster, 2006, 161-180

[11] Magalon, J., Maiers, M., Kurtzberg, J., Navarrete, C., Rubinstein, P., Brown, C., Schramm, C., Larghero, J., Katsahian, S., Chabannon, C., Picard, C., Platz, A., Schmidt, A. \& Katz, G., Banking or Bankrupting: Strategies for Sustaining the Economic Future of Public Cord Blood Banks, PLOS ONE 10 (2015), 1-12

[12] Horwitz, M. \& Chao, N. (Hrsg.), Cord Blood Transplantations, 1st Edition, Springer International Publishing, Cham, 2017

[13] Waldby, C., The Oocyte Economy: The Changing Meaning of Human Eggs, 1st Edition, Duke University Press, Durham, 2019

[14] van de Wiel, L., The Speculative Turn in IVF: Egg Freezing and the Financialization of Fertility, New Genetics and Society 39 (2020), 306-326

[15] Corley-Smith, G. E. \& Brandhorst, B. P., Preservation of Endangered Species and Populations: A Role for Genome Banking, Somatic Cell Cloning, and Androgenesis?, Molecular Reproduction and Development 53 (1999), 363-367

[16] Lanza, R. P., Dresser, B. L. \& Damiani, P., Cloning Noah's Ark, Scientific American 283 (2000), 84-89

[17] Chrulew, M., Freezing the Ark: The Cryopolitics of Endangered Species Preservation, in: J. Radin \& E. Kowal (Hrsg.), Cryopolitics. Frozen Life in a Melting World, Chapt. 14, The MIT Press, Cambridge MA, 2017

[18] van Dooren, T., Flight Ways. Life and Loss at the Edge of Extinction, First Edition, Columbia University Press, New York, 2014

[19] Holmes, D. R. \& Marcus, G. E., Collaboration Today and the Re-Imagination of the Classic Scene of Fieldwork Encounter, Collaborative Anthropologies 1 (2008), 81-101

[20] Westbrook, D. A., Navigators of the Contemporary: Why Ethnography Matters, 1st Edition, University of Chicago Press, Chicago, 2008 